\title{
A Nonparametric Approach to the Removal of Documented Inhomogeneities in Climate Time Series*
}

\author{
Chiara Ambrosino AND Richard E. Chandler \\ Department of Statistical Science, University College London, London, United Kingdom
}

(Manuscript received 22 June 2012, in final form 5 December 2012)

\begin{abstract}
Climate data often suffer from artificial inhomogeneities, resulting from documented or undocumented events. For a time series to be used with confidence in climate analysis, it should only be characterized by variations intrinsic to the climate system. Many methods (e.g., direct or indirect) have been proposed according to the data characteristics (e.g., location, variable, or data completeness). This paper is focused on the abruptchanges problem (when the properties of a time series change abruptly), when their timing is known, and suggests that a nonparametric regression framework provides an appealing way to correct for discontinuities in such a way as to recognize and allow for the existence of other structures such as seasonality and long-term smooth trends. The approach is illustrated by using reanalysis data for southern Africa, for which discontinuities are present because of the introduction of satellite technology in 1979.
\end{abstract}

\section{Introduction}

Reliable and long-term climate records play a crucial role in understanding atmospheric features and identifying their associations with climate variability, extreme events, and long-term trends. Climatic time series often contain discontinuities that are artifacts of the measurement process rather than features of the real climate system, however. A discontinuity of this type may be due to changes in the recording accuracies, instrument change, human or animal interference, variations in the time (e.g., recording hour of the day) of the observation, and changes in the environment of the monitoring station, for example. Those changes can lead to inaccurate or unrealistic time series characteristics and need, therefore, to be accounted for (Peterson et al. 1998; Aguilar et al. 2003; Beaulieu et al. 2007).

As an alternative to station data, reanalysis products are widely used in climate science, commercial, and

\footnotetext{
* Supplemental information related to this paper is available at the Journals Online website: http://dx.doi.org/10.1175/JAMCD-12-0166.s1.

Corresponding author address: Chiara Ambrosino, Dept. of Statistical Science, University College London, 1-19 Torrington Place, London WC1E 6BT, United Kingdom.

E-mail: c.ambrosino@ucl.ac.uk
}

business applications, including in poorly observed areas such as the African continent. As mentioned by Thorne and Vose (2010), reanalysis data have many advantages over other climatic gridded datasets. These advantages include, among others, the use of physical rather than statistical interpolation to produce complete spatial and temporal coverage and the provision of estimates for unobservable quantities, such as potential vorticity. Reanalysis data do have limitations that should be recognized when they are used (Tennant 2004; Sterl 2004).

In reanalysis assimilation, recorded observations from a mix of instruments are used to constrain a numerical weather prediction (NWP) model that is based on physical laws and is run in a hindcast setting. The main goal is the provision of consistent and accurate retrospective records of various climatic variables, currently extending back to the middle or beginning of the twentieth century (Kalnay et al. 1996; Compo et al. 2011). Such an ambitious goal, however, involves limitations related to errors in the assimilated observations, the NWP model, and the methods chosen (Thorne and Vose 2010; Dee et al. 2011).

Indeed, although the data assimilation system is kept unchanged over the reanalysis period, reanalysis data are affected by changes in the observing system and the quality, number, and distribution of the observations in time and space (Bengtsson et al. 2004). Changes in the observation system can result in artificial jumps and 
shifts in the final data product (Dee 2005). Moreover, the introduction of additional observations at any point during the reanalysis process results in less intrinsic variability of the final product, potentially leading to spurious trends (Sterl 2004). In particular, clear continuity problems have been identified following the introduction of satellite data after 1979, notably in the Southern Hemisphere (Sturaro 2003). Discontinuities may therefore be present both in station data and in reanalysis products.

In this article we consider situations in which the time of a potential discontinuity is known. There are many examples of this type, including documented changes of instrumentation (Thompson et al. 2008), documented station moves (Changnon and Kunkel 2006), introduction of new observing data systems (Sturaro 2003), and changes in measurement units (Yang 2006). The detection of undocumented potential discontinuities is much more challenging and is prone to statistical misunderstandings because the usual theory of statistical estimation breaks down in this situation; see Lund and Reeves (2002) for further details.

Several techniques have been proposed in the literature both to test for inhomogeneity in a series that is not caused by climatic factors and to correct for it (Peterson et al. 1998; Aguilar et al. 2003; Ducré-Robitaille et al. 2003; Reeves et al. 2007; Costa and Soares 2009). Those tests differ in the use or not of reference time series for comparison and adjustment, in terms of distribution and trend assumptions (i.e., parametric or nonparametric), number of change points detected (i.e., multiple or single), and whether change point and trends (smooth and/ or abrupt) are or are not estimated simultaneously (Ferguson and Villarini 2012; Bates et al. 2012). Parametric (Buishand 1982; Szentimrey 2003) or nonparametric (Von Neumann 1941; Wald and Wolfowitz 1943; Mann 1945; Kruskal 1952; Pettit 1979; Alexandersson 1986) tests are often used to test for the presence of a break in the data.

A common practice for the correction of inhomogeneities is the use of regression-based approaches in which linear regressions or subset means are fitted before and after the break (Easterling and Peterson 1995). In the presence of additional structures in the series, however, the effect of such methods may not be as intended. Figure 1 shows examples of this phenomenon for time series characterized by a negative (Figs. 1a-c) and positive (Figs. 1d-f) trend. One thousand series were randomly simulated, with the magnitude of the jump known. The same was subsequently estimated (as represented in Fig. 1 by the vertical solid arrows) by using generalized additive models (GAM; Figs. 1a,d; see section 2 for details), subset averages (Figs. 1b,e), and linear regressions (Figs. 1c,f) that are applied pre- and postjump.
The mean-square errors of the estimated jump values for the 1000 simulations were 2.61 and 2.62 (examples in Figs. 1a and 1b), 147.40 and 2086.21 (examples in Figs. 1b and 1e), and 33.96 and 17.89 (examples in Figs. 1c and 1f), indicating that the GAMs give the best estimate of the cycle in this setting. The use of pre- and postjump averages and linear models, on the other hand, resulted in the magnitude of the step change being underestimated in the case of an opposed trend and direction of the jump (Figs. 1b,c) or overestimated (Figs. 1e,f) in the case of the same direction of trend and jump.

The purpose of this note is to highlight the potential for accurate removal of discontinuities using flexible and powerful statistical models that represent explicitly the step change along with other relevant factors (e.g., trend and seasonality) that may account for some of the time series variability. To illustrate the proposed method we consider the detection and removal of discontinuities in reanalysis data arising from this introduction of satellite data. In section 2 the proposed technique is presented. The results of an application are shown in section 3, and the conclusions are presented in section 4 . In addition, the $\mathrm{R}$ software language code file ( $\mathrm{R}$ Development Core Team 2012) used to create Figs. $2 a$ and $2 b$ (described below) is provided in the supplemental material for this paper. It is in text-file form and can be read with a word processor as well as in $\mathrm{R}$.

\section{Methods}

The general requirements to build a statistical model to adjust for discontinuities include the estimation of a trend (defined here as long-term temporal variation in any statistical property), seasonal cycle, and irregular components (Chandler and Scott 2011, pp. 57, 137). If a discontinuity is present, the model can be written as

Series $=$ Trend + Seasonality + Jump + Irregular,

with as many jump indicators as the number of the abrupt changes in the series. After estimating each of those components from the data, the estimated jump contribution can be subtracted and the discontinuity removed.

In climate time series with temporal resolution that is higher than annual, the seasonal cycle is an important factor and can be accounted for by including terms in the model that specifically represent the seasonality in a smooth way. When the seasonality is fairly constant throughout the time series and the annual fluctuations around the mean are reasonably regular, Fourier series of sine and cosine covariates are often used to represent period functions with frequencies that are a multiple of 


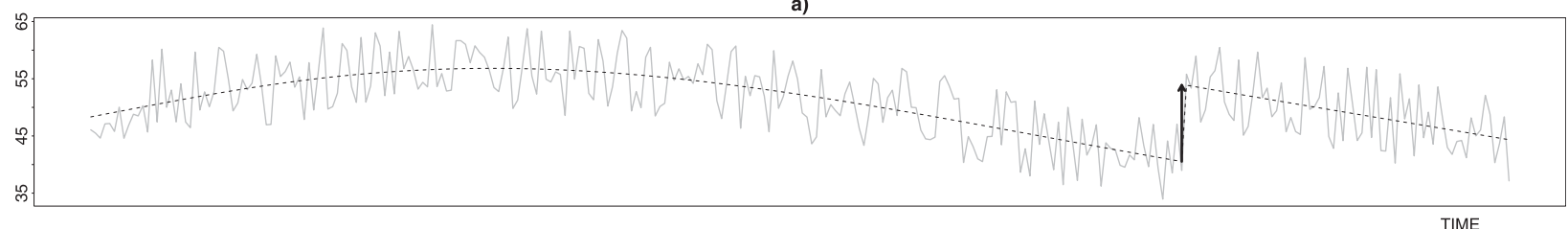

b)

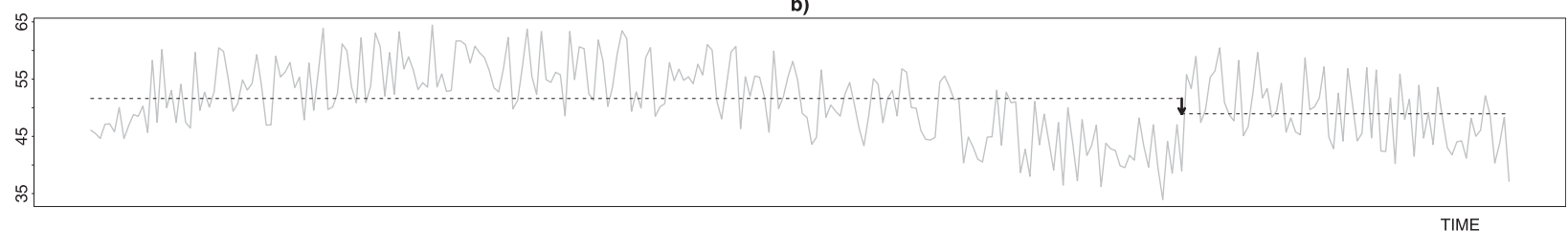

c)

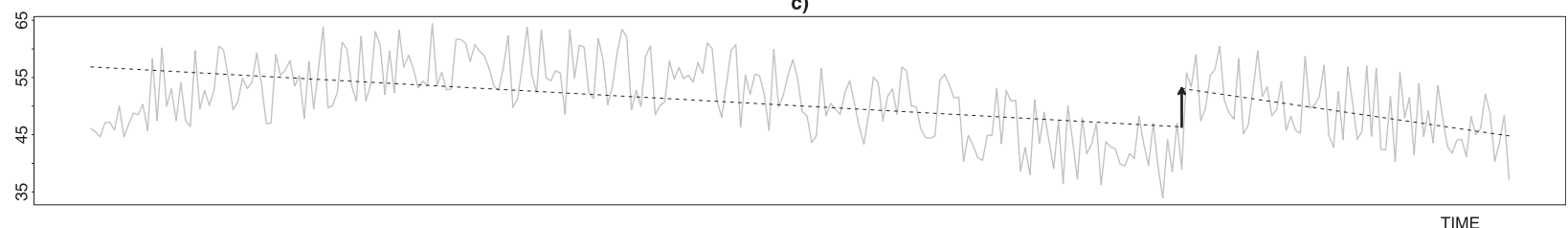

d)

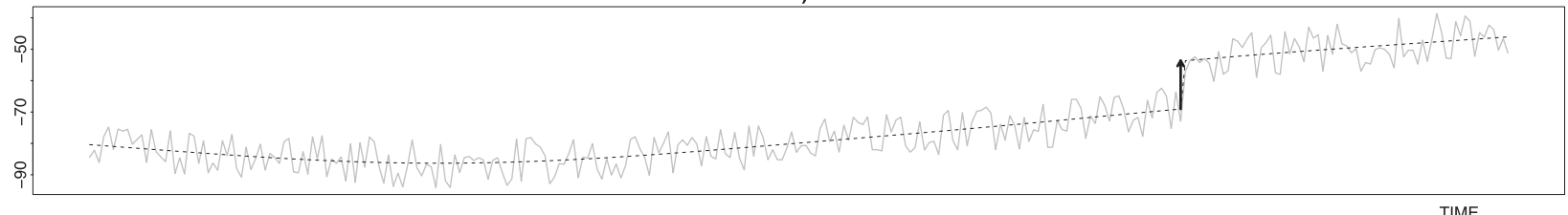

e)

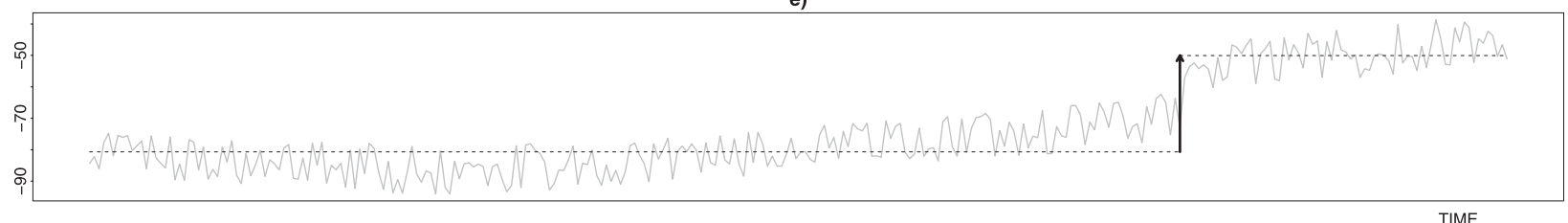

f)

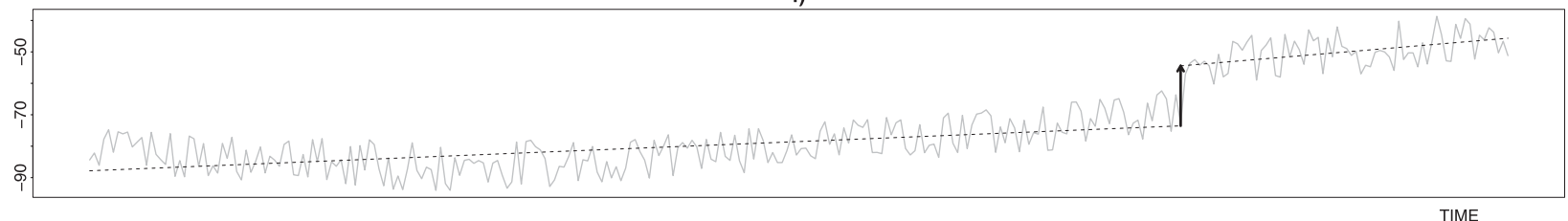

FIG. 1. Magnitude of the step change estimated in series characterized by a (a)-(c) negative and (d)-(f) positive trend. Gray solid lines represent the simulated time series. Dashed lines represent fitted values using a GAM [in (a) and (d)], pre- and postjump averages [in (b) and (e)], and linear trends that are fitted pre- and postjump [in (c) and (f)]. The vertical arrow represents the estimated magnitude of the jump. In (a) and (c) the trend and the direction of the jump are opposed: the magnitude of the step is underestimated in (b) and (c). In (e) and (f) the trend and jump are in the same direction: the magnitude of the step is overestimated.

the annual cycle (Chandler and Scott 2011, p. 90). Alternative ways may be needed to estimate a changing seasonal cycle. In this case, the seasonal cycle for a month could be estimated as a weighted average for the same month in neighboring years (Chandler and Scott 2011, p. 57). Trends can be estimated with parametric or nonparametric techniques. The chosen method should be flexible enough to represent whatever structure is present in the data. In this context, nonparametric approaches provide some advantage because they do not make assumptions about the form of the structure, allowing the data to "speak for themselves" and dictate the form of the trend.

A detrended/deseasonalized/homogeneous series can be derived by subtracting the appropriate component from the representation in Eq. (1). Care does need to be taken when analyzing data that are detrended nonparametrically because the detrending procedure itself 

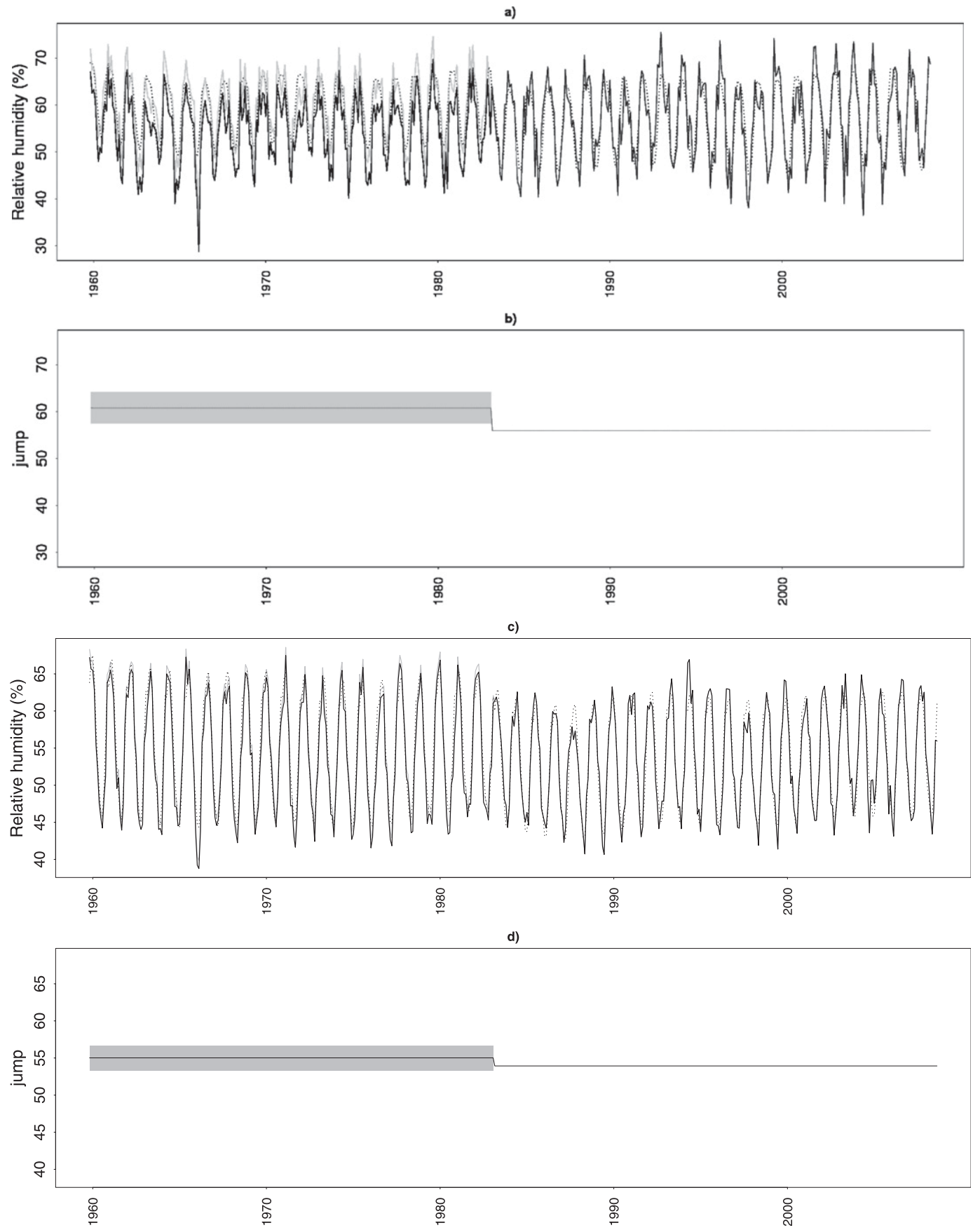

FIG. 2. Illustration of adjustment to NCEP relative humidity time series. (a) Adjusted time series with seasonal cycle for the grid cell with its center at coordinates $25.7^{\circ} \mathrm{S}, 32.5^{\circ} \mathrm{E}$. (b) Uncertainty band of the estimated jump. (c),(d) As in (a) and (b), but for the mean over all grid cells. In each one of the plots, the gray solid line is the original series RHUM $_{t}$, the dashed black line is the estimate of Trend + Jump + Seasonality, and the solid black line is the adjusted series RHUM*

introduces structures that must be accounted for subsequently (Chandler and Scott 2011, p. 54).

To remove the effect of the step change, we used GAMs (Bowman and Azzalini 1997; Wood 2006). GAMs are a nonparametric extension of generalized linear models (GLMs), which themselves extend multiple-linearregression models. As with the GLMs, GAMs allow for different distributions to be fitted to the response 
variables (e.g., normal, exponential, gamma, Poisson, binomial, or inverse Gaussian), accommodating a very wide range of applications. Moreover, smooth functions, rather than just parametric terms, can be added to the model. The literature offers several types of smoothing techniques (e.g., local linear smoothing and spline smoothing). The choice related to the method includes the selection of the smoothing parameter, which refers to size of the response values to be considered. Here, we use cross validation to select an appropriate smoothing parameter; for alternatives, see Bowman and Azzalini (1997) and Chandler and Scott (2011).

Model diagnostics can be performed through a residual analysis. If the model is adequate, a time series plot of residuals should be scattered around zero without any obvious structure. Further examples of residual checks may, for example, include normal quantilequantile plots, autocorrelation function (ACF) of residuals, and plots of residuals versus fitted values. If many time series are to be processed so that it is not feasible to inspect plots for each series individually, aggregated plots can be produced for subsets of residuals to check for spatial and temporal structure (see, e.g., Chandler 2005).

\section{Example}

Here, monthly 850 -hPa relative humidity data from the National Centers for Environmental PredictionNational Center for Atmospheric Research (NCEPNCAR) Reanalysis 1 (Kistler et al. 2001) (NCEP hereinafter) for the period $1958-2001$ and area $12^{\circ}-40^{\circ} \mathrm{S}$ and $0^{\circ}-42^{\circ} \mathrm{E}$ are used to illustrate the method described above. In this time series, a step change is present in 1979 because of the introduction of satellite technology.

At each grid cell, a model of the form

$$
\begin{aligned}
\operatorname{RHUM}_{t}= & a+[b \times I(\text { Year }<1979)] \\
& +s(\text { Month, Year })+\varepsilon_{t}
\end{aligned}
$$

was fitted to the original time series (Ambrosino et al. 2011). Here, the trend term $s$ (Month, Year) is a smooth bivariate function, represented nonparametrically so as to avoid imposing artificial structures that may be unrealistic. By using a bivariate function rather than, for example, a sum of the two univariate functions of the form $s_{1}$ (Month) and $s_{2}$ (Year), we allow for the possibility that the seasonal cycle may change through time (Chandler and Scott 2011). The I(Year < 1979) is the indicator variable of the change point, taking the value 1 for all observations prior to 1979 and 0 thereafter. The

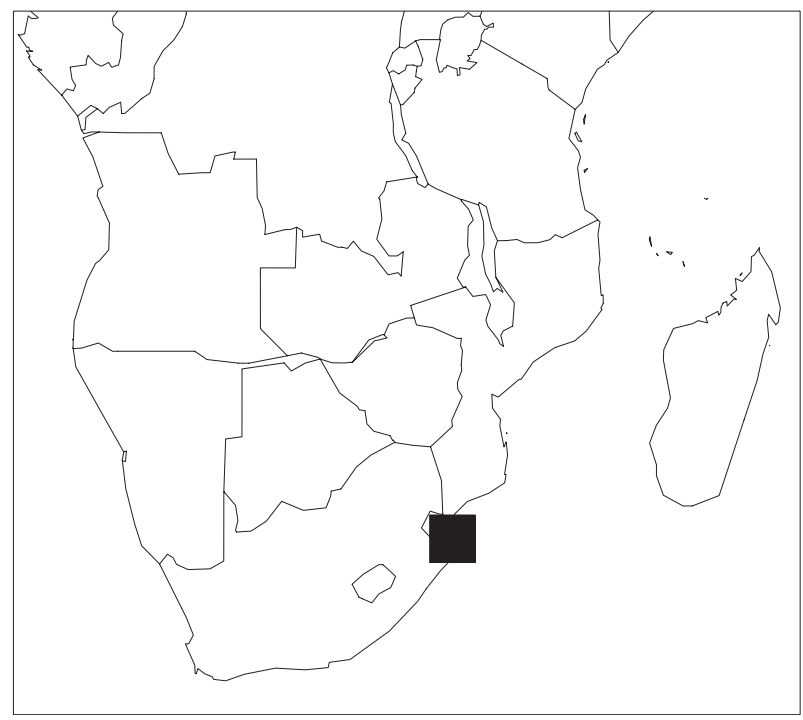

FIG. 3. Location of the selected grid cell for the analysis shown in Figs. 2a and $2 \mathrm{~b}$ and in Fig. 6, described below.

inclusion of an interaction term between the changepoint indicator and the seasonality component would allow for the representation of a changing seasonality concurrent with the jump, if needed.

Estimation of the smooth interaction between the trend and the changing seasonal cycle $s$ (Month, Year), along with the coefficients $a$ and $b$, was carried out using smoothing splines through the "mgcv()" library (Wood 2006) in R, which selects a smoothing parameter automatically by cross validation.

The coefficient $b$ in Eq. (2) is the magnitude of the step associated with the introduction of satellite data. Subtraction of the estimated jump prior to 1979 leaves the values after 1979 unaffected, since those are considered to be more reliable (Tennant 2004). A destepped series can thus be defined as

$$
\mathrm{RHUM}_{t}^{*}=\hat{a}+\hat{s}(\text { Month, Year })+\varepsilon_{t} ;
$$

this series is referred to as "adjusted relative humidity."

An example of the process introduced in the previous section is illustrated in Figs. $2 a$ and $2 b$ (see also the supplemental material) for a single grid cell centered at $32.5^{\circ} \mathrm{S}, 20.0^{\circ} \mathrm{E}$ (see Fig. 3 for its location with respect to southern Africa). Figure 2a shows the original time series $\left(\mathrm{RHUM}_{i}\right.$; solid gray line), the adjusted time series (RHUM ${ }_{t}^{*}$; solid black line), and the estimate of $\hat{a}+\hat{s}$ (Month, Year) (dashed black line) for the NCEP time series. Figure $2 \mathrm{~b}$ shows the confidence interval band of the estimated step change. Figures $2 \mathrm{c}$ and $2 \mathrm{~d}$ are the same as Figs. 2a and $2 b$ but for an averaged time series among all 180 grid cells of the study area. Figure 4 shows separately the time series plotted in Fig. 2a. 

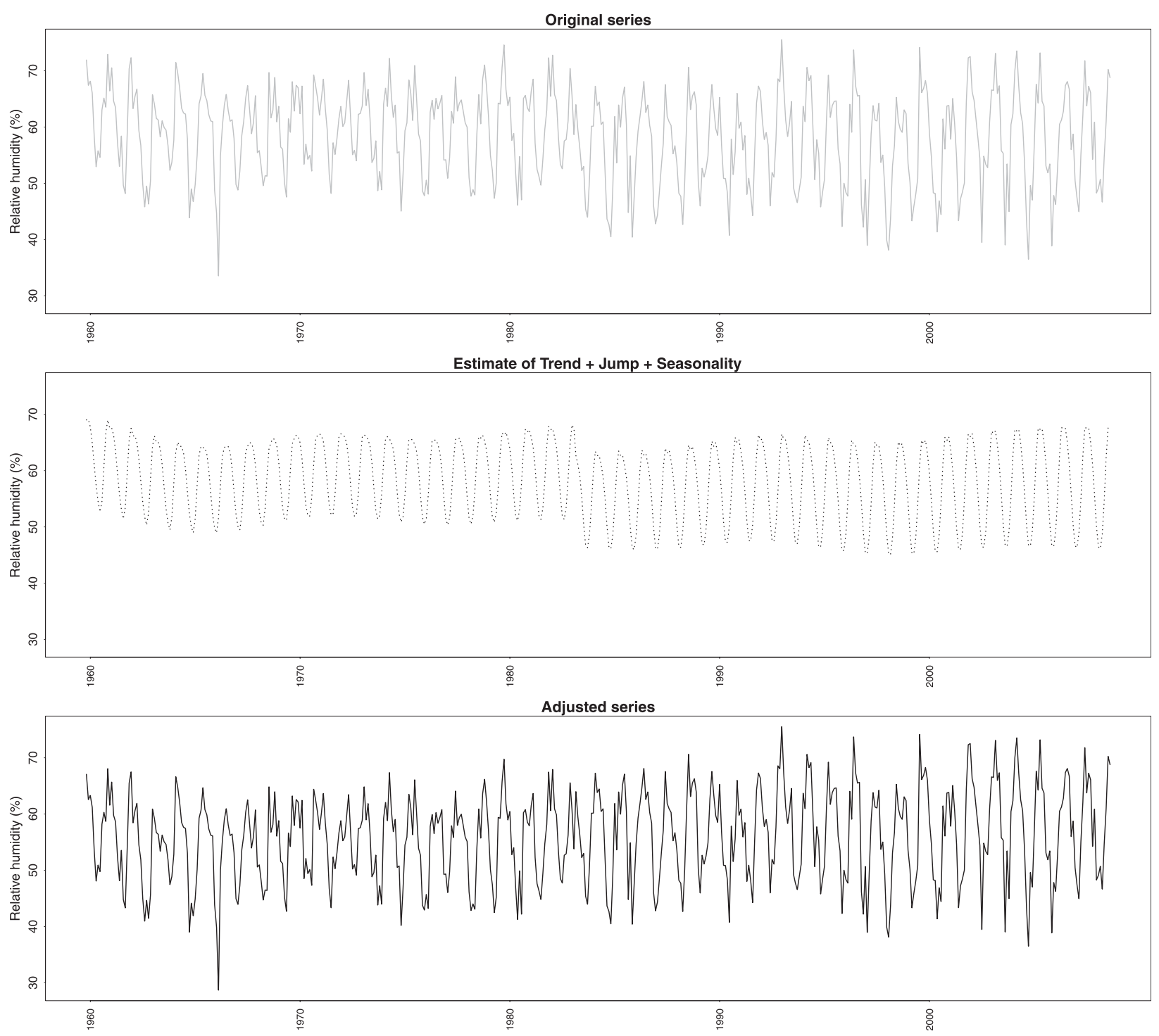

FIG. 4. Decomposition of Fig. 2a for more clarity: (top) the original series RHUM , $_{t}$ (middle) the estimate of Trend + Jump + Seasonality, and (bottom) the adjusted series RHUM

All plots in Fig. 2 show a visible step between the end of 1978 and the beginning of 1979 . The method that was used allows for different magnitudes of step change to be accounted for as well as different amplitudes of the seasonal cycle.

A potential concern with the approach is that if no step change is present then one should not adjust for it. In this case, however, the estimated step change will be 0 to within sampling error and, providing this sampling error is not too large, the adjusted series will be very similar to the original series. Some of the grid cells are indeed characterized by a null step regression coefficient. Where the step change is present, the method allows for the estimation of positive or negative values. Software packages provide information about the level of precision as standard error of the estimate. In the example shown in Fig. $2 b$, the standard error of the estimated jump is around 1.5 units, suggesting that the adjusted series will be accurate within \pm 3 units. This information about the level of uncertainty can be propagated throughout the subsequent analysis, if necessary. Figure 5 shows the estimated step coefficients [ $b$ in Eq. (2)] throughout the study area. Dark-gray shades represent negative steps, and light-gray to white shades represent positive steps. In the southern, central, and northeastern part of the study area the step changes appear to be positive. Conversely, areas above the coast of Angola and Namibia are characterized by a negative step regression coefficient. 


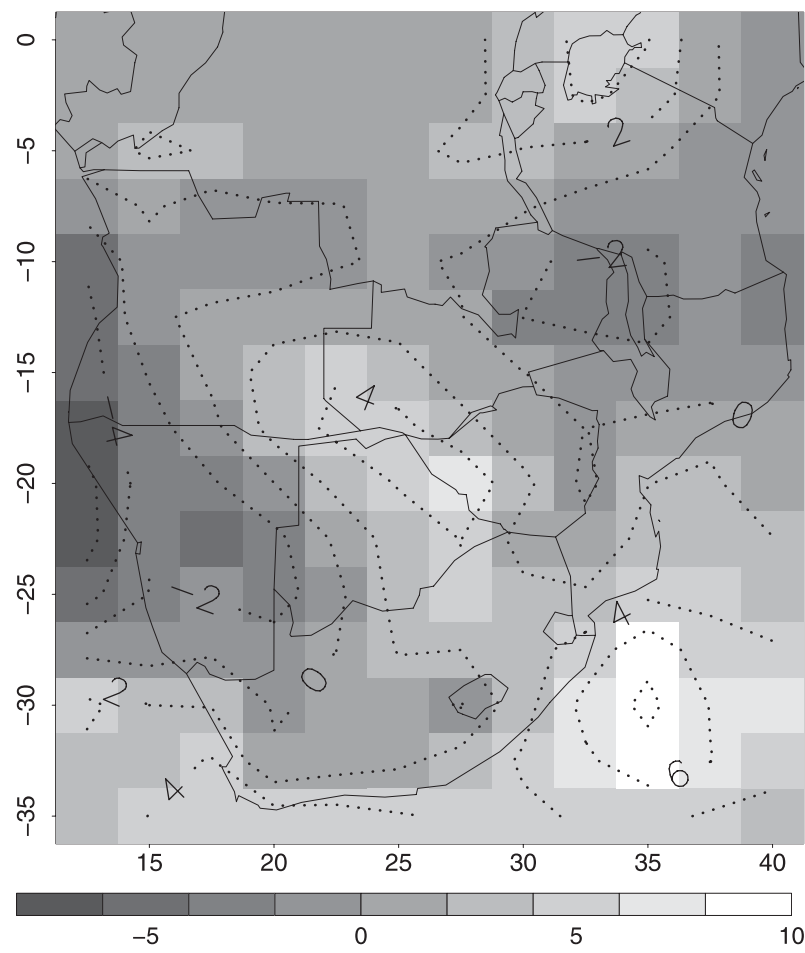

FIG. 5. Estimated step regression coefficients $[b$ in Eq. (2)] for all grid cells in the study area. Contours show the magnitude of the step; white and light-gray shades represent positive values; darker shades of gray represent negative values.

If, in the case of a known step change, the model was to be fitted without a jump indicator, the estimation of the trend component would be spurious. Figure 6 shows that example for the selected grid cell of Fig. 4. The solid black line represents the estimated trend plus changing seasonal cycle where a model was fitted with inclusion of a jump indicator. The dashed gray line represents the estimated trend plus changing seasonal cycle of a model fitted without the jump indicator. Also, residual analysis was performed in terms of normal quantile-quantile plots, residuals versus fitted values, and $\mathrm{ACF}$, and none showed any relevant problem.

\section{Conclusions}

Climate datasets, including reanalysis, are often characterized by inhomogeneities that undermine their suitability in research. Reanalysis datasets are widely used in climate science, and their availability is an important asset, especially in those area characterized by scarcity of data (e.g., Africa). The assimilation of satellite infrared and microwave retrieval into the reanalysis process in 1979 caused a discontinuity in the final reanalysis output, the effect of which is often greater in the Southern Hemisphere (Sturaro 2003; Sterl 2004).

Various techniques have been developed to correct for step changes depending on the nature of the change points and available metadata. This study introduces a simple nonparametric method that is aimed at detecting and adjusting for artificial discontinuities when the times of the potential discontinuities are known. Generalized additive models allow the addition for nonparametric factors as model terms. The advantage of this technique is its flexibility in reproducing smooth trends, meaning the magnitude of the step change could be estimated with less bias in comparison with methods that represent trends with averages or linearly. Generalized additive models were fitted to NCEP reanalysis datasets for a study area in the African continent, and the existence of a step consistent with the 1979 satellite data assimilation was found in the time series. We found that the magnitude of the step varies across the study area, with the highest positive values over the central and northeastern part of the area as well as the southern African coast. The method is able to adjust for those positive, negative, and null trends and could be useful to avoid misjudging the magnitude of trends in longterm climatic data. For detection and correction of undocumented discontinuities, see Lund and Reeves (2002).

Acknowledgments. The lead author is supported by the NERC Changing Water Cycle Programme under

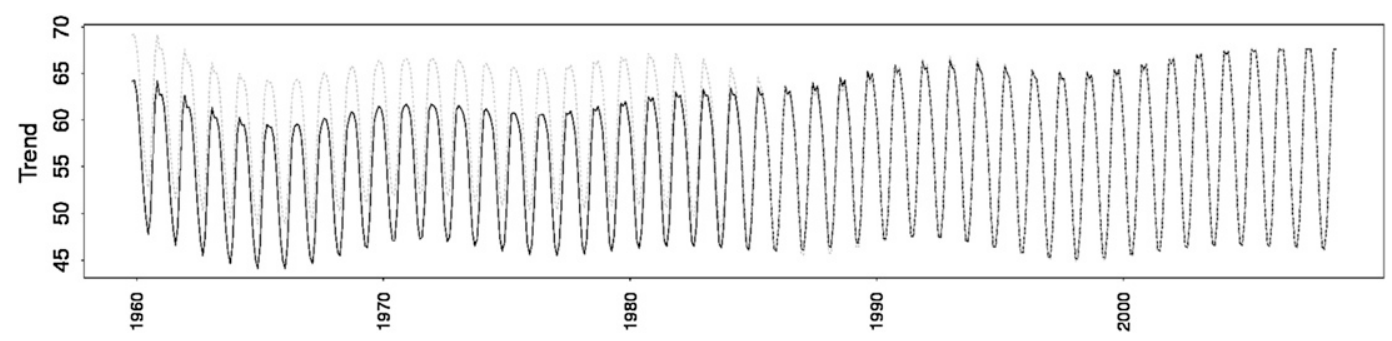

FIG. 6. Estimated trend plus changing seasonal cycle from a model fitted with or without the jump indicator. The solid black line represents the estimated trend plus changing seasonal cycle for a model fitted with a jump indicator. The dashed gray line represents the estimated trend plus changing seasonal cycle for a model fitted without the jump indicator. The time series refers to Fig. 2a. 
Grant NE/I006621/1. Part of the research was funded by the Environment Institute UCL Ph.D. scholarship. The authors thank the reviewers for their comments that helped to improve the manuscript.

\section{REFERENCES}

Aguilar, E., I. Auer, M. Brunet, T. C. Peterson, and J. Wieringa, 2003: Guidance on metadata and homogenization. World Meteorological Organization Tech. Doc. WMO/TD 1186, 50 pp. [Available online at http://www.wmo.int/datastat/documents/ WCDMP-53_1.pdf.]

Alexandersson, H., 1986: A homogeneity test applied to precipitation data. Int. J. Climatol., 6, 661-675.

Ambrosino, C., R. E. Chandler, and M. C. Todd, 2011: Southern African monthly rainfall variability: An analysis based on generalized linear models. J. Climate, 24, 4600-4617.

Bates, B. C., R. E. Chandler, and A. W. Bowman, 2012: Trend estimation and change point detection in climatic series using flexible regression methods. J. Geophys. Res., 117, D16106, doi:10.1029/2011JD017077.

Beaulieu, C., T. B. M. J. Ouarda, and O. Seidou, 2007: Synthèse des techniques d'homogénéisation des séries climatiques et analyse d'applicabilité aux séries de précipitations (Synthesis of homogenization techniques of climate series and analysis of their applicability to precipitation series). Hydrol. Sci. J., 52, $18-37$.

Bengtsson, L., S. Hagemann, and K. Hodges, 2004: Can climate trends be calculated from reanalysis data? J. Geophys. Res., 109, D11111, doi:10.1029/2004JD004536.

Bowman, A., and A. Azzalini, 1997: Applied Smoothing Techniques for Data Analysis: The Kernel Approach with S-Plus Illustrations. Oxford University Press, 193 pp.

Buishand, T. A., 1982: Some methods for testing the homogeneity of rainfall records. J. Hydrol., 58, 11-27.

Chandler, R. E., 2005: On the use of generalized linear models for interpreting climate variability. Environmetrics, 16, 699-715.

- and E. M. Scott, 2011: Statistical Methods for Trend Detection and Analysis in the Environmental Sciences. John Wiley and Sons, 388 pp.

Changnon, S. A., and K. E. Kunkel, 2006: Changes in instruments and sites affecting historical weather records: A case study. J. Atmos. Oceanic Technol., 23, 825-828.

Compo, G. P., and Coauthors, 2011: The Twentieth Century Reanalysis Project. Quart. J. Roy. Meteor. Soc., 137, 1-28.

Costa, A. C., and A. Soares, 2009: Homogenization of climate data: Review and new perspective using geostatistics. Math. Geosci., 41, 291-305.

Dee, D. P., 2005: Bias and data assimilation. Quart. J. Roy. Meteor. Soc., 131, 3323-3343.

— E. Källén, and A. J. Simmons, 2011: Comments on "Reanalysis suitable for characterizing long-term trends." Bull. Amer. Meteor. Soc., 92, 65-70.

Ducré-Robitaille, J.-F., L. A. Vincent, and G. Boulet, 2003: Comparison of techniques for detection of discontinuities in temperature series. Int. J. Climatol., 23, 1087-1101.
Easterling, D. R., and T. C. Peterson, 1995: A new method for detecting undocumented discontinuities in climatological time series. Int. J. Climatol., 15, 369-377.

Ferguson, C. R., and G. Villarini, 2012: Detecting inhomogeneities in the Twentieth Century Reanalysis over the central United States. J. Geophys. Res., 117, D05123, doi:10.1029/2011JD016988.

Kalnay, E., and Coauthors, 1996: The NCEP/NCAR 40-Year Reanalysis Project. Bull. Amer. Meteor. Soc., 77, 437-471.

Kistler, R., and Coauthors, 2001: The NCEP-NCAR 50-Year Reanalysis: Monthly means CD-ROM and documentation. Bull. Amer. Meteor. Soc., 82, 247-268.

Kruskal, W. H., 1952: A nonparametric test for the several sample problem. Ann. Math. Stat., 23, 525-540.

Lund, R., and J. Reeves, 2002: Detection of undocumented changepoints: A revision of the two-phase regression model. J. Climate, 15, 2547-2554.

Mann, H. B., 1945: Non-parametric test against trend. Econometrika, 13, 245-259.

Peterson, T. C., and Coauthors, 1998: Homogeneity adjustments of in situ atmospheric climate data: A review. Int. J. Climatol., 18, 1493-1517.

Pettit, A. N., 1979: A non-parametric approach to the change-point detection. Appl. Stat., 28, 126-135.

R Development Core Team, 2012: R: A Language and Environment for Statistical Computing. Foundation for Statistical Computing, 409 pp. [Available online at http://www.lsw.uni-heidelberg. de/users/christlieb/teaching/UKStaSS10/R-refman.pdf.]

Reeves, J., J. Chen, X. L. Wang, R. Lund, and Q. Lu, 2007: A review and comparison of changepoint detection techniques for climate data. J. Appl. Meteor. Climatol., 46, 900-915.

Sterl, A., 2004: Notes and correspondence on the (in)homogeneity of reanalysis products. J. Climate, 17, 3866-3873.

Sturaro, G., 2003: A closer look at the climatological discontinuities present in the NCEP/NCAR reanalysis temperature due to the introduction of satellite data. Climate Dyn., 21,309-316.

Szentimrey, T., 2003: Statistical problems connected with the homogenization of climatic time series. Climate Dyn., 21, 309-316.

Tennant, W. J., 2004: Considerations when using pre-1979 NCEP/ NCAR reanalyses in the Southern Hemisphere. Geophys. Res. Lett., 31, L11112, doi:10.1029/2004GL019751.

Thompson, D. W., J. J. Kennedy, J. M. Wallace, and P. D. Jones, 2008: A large discontinuity in the mid-twentieth century in observed global-mean surface temperature. Nature, 453, 646-649.

Thorne, P. W., and R. S. Vose, 2010: Reanalysis suitable for longterm trends: Are they really achievable? Bull. Amer. Meteor. Soc., 91, 353-361.

von Neumann, J., 1941: Distribution of the ratio of the mean square successive difference to the variance. Ann. Math. Stat., 14, 367-395.

Wald, A., and J. Wolfowitz, 1943: An exact test for randomness in the non-parametric case based on serial correlation. Ann. Math. Stat., 14, 378-388.

Wood, S. N., 2006: Generalized Additive Models: An Introduction with $R$. Chapman and Hall/CRC Press, 392 pp.

Yang, C., R. E. Chandler, V. S. Isham, and H. S. Wheater, 2006: Quality control for daily observational rainfall series in the UK. Water Environ. J., 20, 185-193. 